\title{
Prostate Acinar Adenocarcinoma, Lymphoepithelioma-Like Variant
}

National Cancer Institute

\section{Source}

National Cancer Institute. Prostate Acinar Adenocarcinoma, Lymphoepithelioma-Like

Variant. NCI Thesaurus. Code C39885.

A variant of prostate carcinoma characterized by the presence of malignant cells forming syncytial patterns and dense lymphocytic infiltrates. 